\title{
The mental representation of spatial descriptions
}

\author{
KANNAN MANI and P. N. JOHNSON-LAIRD \\ Centre for Research on Perception and Cognition, Laboratory of Experimental Psychology \\ University of Sussex, Brighton BN1 9QG, England
}

\begin{abstract}
Two experiments investigated the mental representation of spatial descriptions. In Experiment 1, the subjects classified a series of diagrams, each presented after a spatial description, as either consistent or inconsistent with the description. They were then given an unexpected recognition test of their memory for the descriptions. The subjects remembered the meanings of determinate descriptions very much better than those of grossly indeterminate descriptions; their memory for a description was not reliably affected by whether or not the diagram had been consistent with it. Experiment 2 extended these findings and showed that, although the semantic implications of a determinate description are better remembered than are those of an indeterminate description, the verbatim details of an indeterminate description are easier to recall than are those of a determinate description. The results are taken to imply the existence of two different sorts of encoding: propositional representations that are relatively hard to remember but correspond closely to the sentences in the description, and mental models that are relatively easy to remember but are analogous to spatial arrays and accordingly poor in linguistic detail.
\end{abstract}

There has long been a controversy about the underlying nature of mental representations. Some theorists argue that all information, whether perceptual, linguistic, or imaginal, is encoded in an abstract propositional representation (e.g., Anderson, 1978; Palmer, 1975; Pylyshyn, 1973). Others contend that there are different sorts of representation and that visual images depend on an analogue representation that is distinct from a propositional one (Kosslyn \& Pomerantz, 1977; Paivio, 1971; Shepard, 1978). The purpose of this paper is to report the results of two experiments that examined certain consequences of these two alternative theoretical approaches.

Pylyshyn (1973), echoing many philosophers from Hume onward, has pointed out a major difference between propositional and analogue representations: "It would be quite permissible ... to have a [propositional] mental representation of two objects with a relation between them such as 'besides.' Such a representation need not contain a more specific spatial relation such as 'to the left of' or 'to the right of.' It would seem an unreasonable use of the word 'image' to speak of an image of two objects side by side, without the relation between them being either 'to the left of' or "to the right of" (p. 11). This distinction is useful

This research was carried out as part of a project supported by the Social Science Research Council (Great Britain), to whom we are indebted. We are grateful to Kate Ehrlich and Dave Haw for advice: The design of Experiment 2 arose from an earlier unpublished experiment carried out by Dave Haw. We also thank Larry Barsalou for his comments and for drawing our attention to the study by Barclay. This manuscript was accepted during Dr. Robert G. Crowder's tenure as Editor of this journal. because it has empirical consequences. A direct propositional representation of a sentence such as " $A$ cup is next to a spoon" will take some such form as NEXT (CUP, SPOON), as Kintsch (1974) and others have suggested, although the particular syntactic structure of the representation is not at issue here. The point is that such a representation will be able to handle both determinate (i.e., specific) and indeterminate (i.e., unspecific) relations with equal ease, whereas an internal representation in the form of a model of spatial array will represent determinate relations more readily than indeterminate relations. The only way to form a model of one object beside another that is neutral with respect to left and right would be to construct a set of such analogue representations corresponding to the various possibilities.

Our first experiment was designed to discover whether the determinacy of spatial relations exerts any systematic effects on memory. The representation of a visual stimulus is likely to be closely related in structure to its initial perception and, thus, leave little room for the introduction of indeterminacy. The experiment accordingly made use of verbal descriptions of spatial relations. Such descriptions are usually consistent with any number of trivially different layouts, but they are also easily contrived to be grossly indeterminate. Although a reader normally appears to gain only a general impression from a descriptive passage, a deeper level of comprehension is surely possible. It seems plausible to suppose that most people, including those who are bereft of imagery, can construct an interpretation of discourse that is sufficiently rich to be affected by the determinacy of descriptions. 
In the experiment, the subjects were presented verbal descriptions of the spatial relations among four objects. Half of these descriptions were determinate (e.g., The spoon is behind the knife, The knife is to the right of the plate, The fork is to the left of the plate); half of the descriptions were indeterminate (e.g., The spoon is behind the knife, The knife is to the right of the plate, The fork is to the left of the knife; the relation between the plate and the fork is grossly indeterminate, since the plate could be to the right or to the left of the fork). Each description was followed by a diagram, and the subjects had to decide whether or not it was consistent with the description. Finally, the subjects were given an unexpected recognition test of their ability to recall all the descriptions.

If mental representations are solely propositional, then subjects should remember determinate and indeterminate descriptions equally well, since they each can be directly mapped into such a representation; but, if the descriptions are encoded in an internal spatial array, then subjects should remember determinate descriptions better than indeterminate ones, because the latter require more than one such representation if they are to be accurately encoded.

\section{EXPERIMENT 1}

\section{Method}

The subjects acted as their own controls and received eight determinate and eight indeterminate descriptions presented in random order. Each description consisted of three sentences that together described the spatial layout of four objects. The diagram that was presented after each description was consistent or inconsistent with it, with equal frequencies for the two sorts of description. The subjects' initial task was to decide whether or not the diagram corresponded to the description. This procedure ensured that the subjects made every effort to understand the descriptions. After they had evaluated all the pairs of descriptions and diagrams, their memory for the descriptions was tested in an incidental recognition task. For each of the original trials, subjects were presented four alternative descriptions: the original description, a paraphrase of it, and two confusion items that described a different layout of the four objects in which their positions were also changed relative to each other. One of these confusion items had exactly the same relational terms in it as the original description; the other did not. The subjects had to rank order these four alternatives in terms of their resemblance to the description that they had originally seen. The order of the recognition tests was randomized.

Materials and Procedure. Each description consisted of three sentences, and in each sentence the relation between two objects was described. The relation was always one of the following: (1) is to the left of, (2) is to the right of, (3) is in front of, (4) is behind. We constructed eight determinate descriptions, two for each of the four configurations that can be obtained by rotating an "L-shape" through $90 \mathrm{deg}: L, \Gamma, \neg$,$\lrcorner . The frequencies of$ the different relational expressions were balanced overall. The indeterminate descriptions were constructed by modifying the determinate descriptions, although maintaining the same relational expressions within them. For example, the following format for a determinate description

$A$ is behind $D$.

$A$ is to the left of $B$.

$\mathrm{C}$ is to the right of $\mathrm{B}$. is consistent with the layout

$$
\begin{array}{lll}
\text { A } & \text { B } & \text { C } \\
\dot{D} & &
\end{array}
$$

since each object is specified in terms of its relation to an adjacent object. The corresponding indeterminate description,

$A$ is behind $D$.

$A$ is to the left of $B$.

$C$ is to the right of $A$.

differs only in the choice of the last noun phrase, but it is consistent with either of the layouts

$$
\begin{array}{llllll}
\text { A } & \text { B } & \text { C } & \text { A } & \text { C } & \text { B } \\
\text { D } & & & \text { D } & &
\end{array}
$$

since it does not directly specify the relation between B and C. Sixteen different sets of four lexical items were allocated at random to the descriptions. Each such set of terms denoted familiar objects drawn from the same general semantic category.

The diagrams consisted of the printed names of the objects arranged in an appropriate (or inappropriate) configuration, such as,

$$
\begin{aligned}
& \text { bed } \\
& \text { table } \\
& \text { chair bookshelf }
\end{aligned}
$$

The diagrams that were consistent with the descriptions were always in the form of an L-shape or one of its rotations, with each of the four possibilities occurring equally often. The diagrams that were inconsistent with the descriptions contained the appropriate objects in a different L-shape and in different relative positions to each other. The same diagram (apart from the necessary changes in lexical content) was used for a determinate description and its corresponding indeterminate description.

The four descriptions in each recognition test comprised the original description, a close paraphrase of it obtained by expressing the converse relations for two of the sentences (e.g., " $A$ is to the left of $B$ " was replaced by " $B$ is to the right of $A "$ ), and two confusion items that described the objects arranged in different positions relative to each other and in a different rotation of the L-shape. One confusion item contained the same relational expressions in the same order as in the original description, and the other made use of different relational expressions. The confusion items were accordingly radically inconsistent with the original descriptions, whether determinate or indeterminate. The confusion items for the latter, however, were indeterminate, too, as is illustrated in the following example of the four items used in the test for one of the indeterminate descriptions:

\section{Original}

The bookshelf is to the right of the chair.

The chair is in front of the table.

The bed is behind the chair.

\section{Paraphrase}

The bookshelf is to the right of the chair.

The table is behind the chair.

The chair is in front of the bed.

\section{First Confusion Item}

The bookshelf is to the right of the table.

The chair is in front of the table.

The table is behind the bed. 


\section{Second Confusion Item}

The bookshelf is to the right of the table.

The table is behind the chair.

The bed is in front of the table.

The subjects were tested individually. They were told the general nature of their task, which was to decide whether or not a diagram was consistent with a description. When they understood the instructions, they were presented the series of descriptions and diagrams. They were allowed to study a description for as long as they liked. It was removed and the diagram was presented. The subjects again took as long as they needed in order to make their decision: They were not told whether or not they were correct. After the 16 trials, the subjects were given the unexpected recognition task. They were told to put the four alternative descriptions used in each test into the order in which they resembled the original description, and they were explicitly instructed to try to recall the exact wording of the original. The four alternative descriptions used in each test were put in front of them in a random order. There was no time limit in which to carry out the task.

Subjects. Eighteen unpaid volunteers, who were undergraduates at the University of Sussex, took part in the experiment.

\section{Results and Discussion}

An accurate memory for the meaning of a description consists of ranking both the original and its paraphrase as closer to the original than either of the two confusion items. The mean numbers of trials on which this correct performance occurred are presented in Table 1 as a function of the determinacy of the descriptions and the appropriateness of the diagrams. It is evident that determinate descriptions (a mean of 5.6 of 8 ) were much better recalled than indeterminate descriptions (a mean of only 3.1 of 8 ). This difference was extremely reliable: No subjects yielded contrary results, although there were two ties $\left(p=.5^{16}\right)$. We also carried out an analysis of the correlation between the complete rankings of all four recognition items and the correct order: original, paraphrase, confusion item with the same relational terms as the original, and confusion item with relational terms different from those of the original. Performance was scored by assessing the correlation between each trial and the correct rank order, using a simple statistic, $\mathbf{P}$, which is calculated in determining Kendall's rank correlation, $\tau$. The values of $\mathrm{P}$ for four items vary between 6 for a perfect correlation and 0 for a perfect inverse correlation, in which items are ranked in exactly the order opposite to the correct one. The mean P scores

Table 1

Mean Numbers of Trials (of Four) on Which Subjects Ranked the Original Description and Its Paraphrase Higher Than the Two Confusion Items in Experiment 1

\begin{tabular}{ccc}
\hline & \multicolumn{2}{c}{ Description } \\
\cline { 3 - 3 } Diagram & $\mathrm{D}$ & $\mathrm{I}$ \\
\hline Consistent With Description & 3.0 & 1.5 \\
Inconsistent With Description & 2.6 & 1.6 \\
\hline
\end{tabular}

Note $-D=$ determinate $;=$ indeterminate. were 4.5 for the determinate descriptions and 3.4 for the indeterminate descriptions, and the difference was highly reliable (Wilcoxon's $\mathrm{T}=4, \mathrm{n}=18, \mathrm{p}<.0002$ ). The main purpose of this analysis was to use all the data in checking stringently whether there was any effect of the consistency or inconsistency of the diagram with a description on memory for that description. It might be argued that when a diagram is consistent with a description, there is an additional cue to aid memory for the description. Such an effect might perhaps be a case of "congruity" enhancing recall (see Craik \& Tulving, 1975). In fact, however, there was no reliable effect of the nature of the diagram on performance in the recognition task. The $\mathbf{P}$ scores for the determinate descriptions were 4.8 for diagrams that were consistent with the description and 4.2 for diagrams that were inconsistent with the description (Wilcoxon's $\mathrm{T}=46.5$, $\mathrm{n}=18, \mathrm{n} . \mathrm{s}$.); the $\mathrm{P}$ scores for the indeterminate descriptions were 3.4 for diagrams that were consistent with the description and 3.5 for diagrams that were inconsistent (Wilcoxon's $\mathrm{T}=82.5$, n.s.).

Errors in classifying the diagrams as consistent or inconsistent with the descriptions constituted $7.3 \%$ of the responses. The errors did not differ significantly as a function of the nature of the descriptions or diagrams.

The results show clearly that after a classification task, determinate descriptions are better remembered than are indeterminate descriptions. Since a theory based on propositional representations would predict that both would be handled with equal facility, we conclude that subjects are not using such representations for both sorts of descriptions. A clue to the nature of their representations is provided by considering their verbatim recognition of the original descriptions. The mean numbers of trials on which the original verbatim descriptions were ranked prior to the paraphrase descriptions were 4.4 for the determinate descriptions and 4.5 for the indeterminate descriptions. These results obviously do not conform to the difference in the memorability of the meanings of the two sorts of description. We therefore looked at what happened on those trials on which the subjects recalled the meaning of a description correctly (i.e., correctly ranked the original and paraphrase prior to the confusion items), and we examined the likelihood that they selected the original description prior to the paraphrase description. The mean proportions of this conditional verbatim recall were .57 for the determinate descriptions, but .63 for the indeterminate problems. Although the difference was not significant (Wilcoxon's $\mathrm{T}=37.5$, $n=14, p>.05$ ), only in the case of the indeterminate descriptions was the proportion significantly higher than would be expected by chance (indeterminate condition, Wilcoxon's $\mathrm{T}=13, \mathrm{n}=15, \mathrm{p}<.01$; determinate condition, Wilcoxon's $T=30, n=13$, n.s.).

In short, our subjects remembered the meaning of the determinate descriptions very much better than they 
remembered the meaning of the indeterminate descriptions, but the ability to make a verbatim recall did not conform to this trend. Indeed, only the performance with the indeterminate descriptions was significantly better than chance. This pattern of results suggests that the two kinds of description tend to be mentally represented in different ways. The second experiment was designed to elucidate the nature of this difference.

\section{EXPERIMENT 2}

\section{Method}

The design was similar to that of the first experiment, but with one crucial modification: The recognition test contained a description that was not a paraphrase of the original but that could be inferred from the spatial layout corresponding to it. The subjects were presented eight verbal descriptions of the spatial relations between sets of five objects. Each description consisted of four sentences. As in the previous experiment, half the descriptions were determinate and half were indeterminate, and half the diagrams were consistent with the previously presented description and half were inconsistent with it. The order of presenting these trials was random. The determinate descriptions characterized such arrangements as

$$
\left.\prod_{D}^{A-B}\right|^{B}-C
$$

in which the lines are between those items specifically linked by one of the sentences in the description. The indeterminate descriptions were consistent with more than one layout, such as
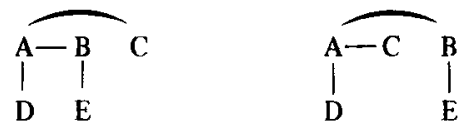

in which the lines are between those items specifically linked by one of the sentences in the description. After each description and diagram had been evaluated, the subjects received an unexpected series of recognition tests, which were also presented in a random order. In each test, the subjects had to rank order four alternative descriptions in terms of the descriptions' resemblance to the description they had actually seen. These four alternatives consisted of the original description, a description that could be only inferred from the spatial layout, and two confusion items, one with the same spatial expressions as the original and one with different spatial expressions. The inferential description contained two of the original sentences, one sentence that was a paraphrase of the corresponding original formed by using the converse relation and one sentence that interrelated two items that had not been explicitly related in the original but could be inferred from a layout consistent with the original description. For example, in the case of the layouts above, the sentence was of the form "D is to the left of $E$." The reader should note that this assertion does not correspond to any sentence in the original description, but it is inferrable from a symmetric layout constructed in accordance to the original. It is true of both the diagrams that can be formed from the indeterminate description. We have here assumed that subjects are likely to form symmetrical mental models of spatial relations, so that $D$ will indeed be roughly the same distance from $A$ that $E$ is from B; people certainly draw symmetrical diagrams for such descriptions (Ehrlich \& Johnson-Laird, Note 1). If they form grossly asymmetrical mental models, then obviously they may be reluctant to infer that $D$ is to the left of $E$.
Our hypothesis was that subjects would construct a mental model of the spatial layout characterized by a determinate description, but that they would abandon the attempt to do so in interpreting an indeterminate description as soon as they encountered the indeterminacy and, instead, try to remember the sentences in the description. The advantage of such a propositional representation is that it would obviate the need to construct two alternative mental models, which would otherwise be necessary in order to ensure a correct classification of the subsequent diagram. We predicted that the subjects' memory for the layout would be better for the determinate than for the indeterminate descriptions, that they would tend to confuse the original and the inferential descriptions in the determinate cases, and that their relative ability to make a verbatim recall would be better for indeterminate descriptions than for determinate descriptions, since a propositional representation is close to the linguistic input.

Materials and Procedure. The materials were similar to those of the previous experiment, except that there were four sentences in each description. We constructed four determinate and four indeterminate descriptions, one for each of the four $90-\mathrm{deg}$ rotations of an "F-shape." The frequencies of the different relational expressions in the descriptions were balanced overall. Each indeterminate description was obtained from a determinate description by changing the allocation of one noun phrase in the second sentence. Thus, the following determinate format

$A$ is to the left of $B$.

$C$ is to the right of $B$.

$D$ is in front of $A$.

$E$ is in front of $B$.

was matched by a corresponding indeterminate description with the same relational terms:
$A$ is to the left of $B$.
$\mathrm{C}$ is to the right of $\mathrm{A}$.
$D$ is in front of $A$.
$\mathrm{E}$ is in front of $\mathrm{B}$.

Eight different sets of five lexical items were allocated at random to the descriptions. Each such set denoted familiar objects drawn from the same general semantic category: We simply added a fifth item to eight of the categories used in Experiment 1.

The diagrams consisted of the typed names of the objects arranged in an appropriate (or inappropriate) configuration. The diagrams that were inconsistent with the descriptions contained the right names in the wrong layout, a different assignment of the objects to their places and a different rotation of the $F$-shape. The same diagram (apart from necessary changes in lexical content) was used for a determinate description and its corresponding indeterminate description.

The four descriptions in each recognition test were constructed along the same general lines as before, except for the inferrable description, which contained two of the original sentences, one converse sentence, and one sentence that correctly characterized the layout, although neither it nor any paraphrase of it had occurred in the original sentences.

The subjects were tested individually, using a procedure that was identical to the one used in Experiment 1.

Subjects. Twenty volunteers, who were undergraduates at the University of Sussex, were paid $£ 1$ for taking part in the experiment. They had not previously participated in any experiment of this kind.

\section{Results}

The mean numbers of trials on which the original description and the inferrable description were ranked 
Table 2

Mean Numbers of Trials (of Two) on Which Subjects Ranked the Original Description and the Inferrable Description Higher Than the Two Confusion Items in Experiment 2

\begin{tabular}{ccc}
\hline & \multicolumn{2}{c}{ Description } \\
\cline { 2 - 3 } Diagram & $\mathrm{D}$ & $\mathrm{I}$ \\
\hline Consistent With Description & 1.9 & 1.0 \\
Inconsistent With Description & 1.7 & 1.3 \\
\hline
\end{tabular}

Note $-D=$ determinate $;=$ indeterminate .

higher than the confusion items were 3.5 out of 4 of the determinate descriptions and 2.3 of 4 of the indeterminate descriptions. Not a single subject went against this trend $\left(\mathrm{p}=.5^{20}\right)$. The mean $\mathrm{P}$ score for determinate descriptions was 4.7 , and the mean $P$ score for the indeterminate descriptions was 4.3 ; the difference between the two conditions was reliable (Wilcoxon's $\mathrm{T}=9, \mathrm{n}=13, \mathrm{p}<.005$ ). Table 2 presents the complete results as a function of both the type of description and the type of diagram. There were no reliable effects of whether or not the diagram was consistent with the description.

The mean numbers of trials on which the original verbatim descriptions were ranked prior to the inferrable descriptions were 2.7 for the determinate descriptions and 3.5 for the indeterminate descriptions. The difference between the two conditions was reliable (Wilcoxon's $\mathrm{T}=9, \mathrm{n}=13, \mathrm{p}<.005$ ). However, there is perhaps some evidence for a slight retention of the original determinate descriptions. In principle, the chance probability of making a completely correct rank ordering of all four items in a recognition test is .04 , although this estimate assumes, perhaps implausibly, that all orderings are equally likely. Only 2 of 20 subjects failed to make at least one correct ranking for determinate descriptions.

Errors in classifying the diagrams constituted $11.25 \%$ of the responses. The errors did not differ significantly as a function of the nature of the descriptions or the diagrams: The mean $P$ scores for trials in which there was an error in classifying the diagram were 3.7 for the determinate condition and 3.6 for the indeterminate condition.

\section{GENERAL DISCUSSION}

Our experiments have shown that subjects tend to remember the gist of determinate descriptions better than the gist of indeterminate descriptions. However, the verbatim detail of sentences is more likely to be remembered from an indeterminate description than from a determinate discription. A plausible explanation of this pattern of results rests on three considerations. First, the initial stage of understanding a sentence consists of forming a relatively superficial linguistic or propositional representation that is close to the surface form of the sentence, a hypothesis that has been advanced by both Fodor, Fodor, and Garrett (1975) and Kintsch (1974). This propositional representation therefore encodes sufficient information to enable verbatim information to be recalled. Second, there is an option to use this propositional representation as the basis for the construction of a mental model whose structure is analogous to a percept of a spatial layout consistent with the description. Hence, the recovery of a propositional representation is a necessary precursor to the construction of a mental model, and the encoding of a spatial description in the form of a model necessarily requires more processing than merely encoding it propositionally. Moreover, once a model has been formed, the propositional representation on which it is based is likely to be discarded. Third, since a greater depth of processing (e.g., Craik \& Lockhart, 1972) or a greater amount of processing makes for enhanced memorability (e.g., Johnson-Laird \& Bethell-Fox, 1978), a mental model should be easier to remember than a propositional representation. We can accordingly distinguish two modes of encoding: mental models, which are easier to remember but contain no information about the specific sentences on which they are based, and propositional representations, which are harder to remember but do distinguish between such assertions as " $\mathrm{A}$ is to the right of $\mathrm{B}$ " and "B is to the left of A." What the findings imply, of course, is that the subjects tended to construct mental models of the determinate premises, but propositional representations of the indeterminate premises. It is difficult to see how else to account for the greater memorability of determinate descriptions, the reliable tendency to confuse them with what can be inferred from them, and the superior recognition of the verbatim indeterminate descriptions.

Could it be that determinate descriptions are better remembered, not because they are represented by models, but because they describe more organized patterns, or are less complex informationally, or require a less abstract degree of representation? Insofar as such explanations can be made clear and objective alternatives, so that they could, for example, be modeled like the present theory in the form of a computer program, or yield different predictions, we believe there are grounds for rejecting each of them. The determinate and indeterminate descriptions differ only in a single word. Whether one is measuring it statistically or semantically (see Bar-Hillel \& Carnap, 1964), a determinate description conveys more information than an indeterminate description does. Such variables as organization and abstractness can come into play only if one abandons a purely linguistic representation, and the only practical way to discover that a description is indeterminate (or determinate) is to try to build a model of it. The point to be emphasized, however, is that the results from the two sorts of description cross over, depending on whether one is measuring gist or verbatim recall. This finding cannot be accommodated by any explanation that resorts to only a single unitary notion, such as 
degree of organization, amount of information, or abstractness of representation.

Granted the need to postulate at least two sorts of representation, it does not follow immediately that one must be a spatial model and the other a propositional representation close to the linguistic form of the description. However, the results of the present experiments thrust such a conclusion on us.

First, the recall of verbatim detail demands access to linguistic form. Second, confusion of the original description with one that can only be inferred from a layout constructed in a symmetrical way from the original demands access to a spatial model.

The theory that we are advocating was originally sketched by Johnson-Laird (1970), who argued that connected discourse is encoded, not primarily in a linguistic form but in a representation similar to a model based on perceiving or imagining the events instead of merely reading or hearing about them. The process of construction was presumed to be based on the meanings of sentences, but also to involve inferences that go beyond them. Bransford, Barclay, and Franks (1972) advanced a similar hypothesis, distinguishing between an "interpretive" and a "constructive" approach to semantics. An interpretive theory assumes that the semantic interpretation assigned to a sentence provides a full analysis of its cognitive meaning. The constructive theory that these authors advocate, however, postulates that subjects construct semantic descriptions of situations that go beyond the linguistically given information. Barclay (1973) illustrated the contrast by presenting to two groups of subjects sentences that described the serial order of five animals standing in a row. One group was told to work out the order, and the other group, which was not told that the sentences described a serial order, was instructed merely to memorize the sentences. In a recognition test, the first group did not distinguish between the original sentences and other sentences that were true of the array; the memorizers, however, were able to discriminate only between those sentences that introduced items not originally interrelated and the remaining sentences in the test. Other work on descriptions of linear orderings has yielded comparable results: With determinate orders, performance with pairs remote from each other is superior to that with adjacent pairs, which suggests that subjects construct a linear array, but the effect disappears in the case of partial orderings, which suggests that they are represented in a different way (see, e.g., Moeser \& Tarrant, 1977; but cf. also Lawson, 1977; Warner \& Griggs, in press).

Why should there be at least two modes of represen. tation? One answer is suggested by the computer program that models our theory of spatial inference. The program builds up an internal spatial array as a function of the propositional representations of the premises presented to it, but it keeps a record of these propositional representations in order to cope with indeter. minacies. On the one hand, the use of internal models simplifies the representation of meanings and facilitates the implementation of inferential heuristics. The meaning of an expression such as " $\mathrm{A}$ is on the right of B" simply requires a specification of the region in which A should be located given the location of B, and, as in the program, this specification can be used both for constructing models and for evaluating sentences with respect to them. Such an account of how an expression relates to a model of the world is a vital part of semantics, but it has been neglected by many psychological theories of meaning. Moreover, given such an account, it is no longer necessary to spell out the logical properties of words in rules of inference or meaning postulates: These properties emerge directly from the basic semantics (see Johnson-Laird, 1980, for further arguments in support of this general claim). On the other hand, ordinary language is so indeterminate that any system that could interpret it only by setting up mental models would be at a serious disadvantage: Either it would be overwhelmed by a combinatorial explosion of alternative models for the same discourse or it would have to embody arbitrary, and often erroneous, assumptions within its models. Hence, there is a need for propositional representations that encode indeterminacies as readily as they encode determinate relations.

\section{REFERENCE NOTE}

1. Ehrlich, K., \& Johnson-Laird, P. N. Spatial descriptions and referential continuity. Journal of Verbal Learning and Verbal Behavior, in press.

\section{REFERENCES}

Anderson, J. R. Arguments concerning representations for mental imagery. Psychological Review, 1978, 85, 249-277.

BARCLAY, J. R. The role of comprehension in remembering sentences. Cognitive Psychology, 1973, 4, 229-254.

Bar-Hillel, Y., \& CarNaP, R. An outline of a theory of semantic information. In Y. Bar-Hillel (Ed.), Language and information. Reading, Mass: Addison-Wesley, 1964.

Bransford, J. D., Barclay, J. R., \& Franks, J. J. Sentence memory: A constructive versus interpretive approach. Cognitive Psychology, 1972, 3, 193-209.

Craik, F. I. M., \& Lockhart, R. S. Levels of processing: A framework for memory research. Journal of Verbal Learning and Verbal Behavior, 1972, 11, 671-684.

Craik, F. I. M., \& Tulving, E. Depth of processing and the retention of words in episodic memory. Journal of Experimental Psychology: General, 1975, 104, 268-294.

Fodor, J. D., Fodor, J. A., \& GarretT, M. F. The psychological unreality of semantic representations. Linguistic Inquiry, 1975, 4, 515-531.

Johnson-Laird, P. N. The perception and memory of sentences. In J. Lyons (Ed.), New horizons in linguistics. Harmondsworth, England: Penguin, 1970.

Johnson-LaInd, P. N. Mental models in cognitive science. Cognitive Science, 1980, 4, 71-115.

Johnson-Laird, P. N., \& Bethell-Fox, C. E. Memory for questions and amount of processing. Memory \& Cognition, $1978,6,496-501$.

Kintsch, W. The representation of meaning in memory. Hillsdale, N.J: Erlbaum, 1974.

Kosslyn, S. M., \& Pomerantz, J. R. Imagery, propositions and 
the form of internal representations. Cognitive Psychology, 1977, 9, 52-76.

LAwSON, R. Representation of individual sentences and holistic ideas. Journal of Experimental Psychology: Human Learning and Memory, 1977, 3, 1-9.

Moeser, S. D., \& Tarrant, B. L. Learning a network of comparisons. Journal of Experimental Psychology: Human Learning and Memory, 1977, 3, 643-659.

Paivio, A. Imagery and verbal processes. New York: Holt, Rinehart, \& Winston, 1971.

Palmer, S. E. Visual perception and world knowledge: Notes on a model of sensory-cognitive interaction. In D. A. Norman, D. E. Rumelhart, \& the LNR Research Group (Eds.), Explorations in cognition. San Francisco: Freeman, 1975.
Pylyshyn, Z. W. What the mind's eye tells the mind's brain: A critique of mental imagery. Psychological Bulletin, 1973, 80, $1-24$.

She Pard, R. N. The mental image. American Psychologist, 1978, 33, 125-137.

Warner, S. A., \& Griggs, R. A. Processing partially ordered information. Journal of Experimental Psychology: Human Learning and Memory, in press.

(Received for publication June 10, 1980; revision accepted October 16,1980 .) 